\title{
Federico Pous, Alejandro Quin y Marcelino Viera (eds.), Autoritarismo, historia cultural y resistencia política en América Latina: exponiendo Paraguay. Cham, Palgrave Macmillan, 2018, 301 páginas
}

\author{
Camilo Caballero Ocariz
}

Cita sugerida: Caballero Ocariz, C. (2020). [Revisión del libro Autoritarismo, historia cultural y resistencia política en América Latina: exponiendo Paraguay por F. Pous, A. Quin y M. Viera (Eds.)]. Orbis Tertius, 25(32), e184.

https://doi.org/10.24215/18517811e184

Comprender y explicar a Paraguay desde los estudios culturales y sociales ha resultado siempre un desafío para los propios paraguayos y también para los no paraguayos. Poco conocido, poco estudiado, frecuentemente excusado en particularidades y frecuentemente estudiado desde adentro, Paraguay encuentra esperanzas de una mayor comprensión internacional en esta obra de alta relevancia para los estudios paraguayos. Esclarecedor para extranjeros, revelador e interpelante para paraguayos, no sería exagerado considerar que para estos resulta una lectura obligatoria. Esclarecedor para extranjeros por su gran aporte académico fundamentado en ser una publicación en ingles que contiene a autores locales, regionales e internacionales, relevador e interpelante para paraguayos fundamentándose en la propuesta marcadamente novedosa de basar la exposición sobre Paraguay incluyendo a literatura clásica, revisión histórica y sobre todo películas contemporáneas conocidas por el público paraguayo. Authoritarianism, cultural history and politica lresistance in Latin America. Exposing Paraguay, o bien simplemente Exponiendo Paraguay como será llamado de aquí en adelante, ofrece exactamente lo que promete: un viaje iluminador sobre la cultura paraguaya y la historia cultural del Paraguay. Una lectura apasionante que luego de evidenciar los sutiles pero penetrantes rasgos culturales autoritarios del Paraguay, dejará al lector con preguntas sobre las posibilidades de modernización cultural en el país y, de paso, se cuenta con un didáctico acercamiento a los estudios culturales. Para culminar la brillantez, el trabajo resalta la expresión "realidad que delira" como una herramienta para pensar el Paraguay. 
La obra, que puede establecer un dialogo productivo con los estudios sobre Paraguay en Argentina, va Exponiendo Paraguay con una innovadora propuesta, que combina análisis de literaturas clásicas del Paraguay, películas contemporáneas de Paraguay, arte, historia, e historia política reciente, cuestiones que en otros estudios sobre Paraguay fueron frecuentemente tratados de manera desconectada, en esta obra se los combina bajo la mirada de los estudios culturales. Dentro del conocido debate sobre los idiomas de las publicaciones científicas, Exponiendo Paraguay es una decidida apuesta por cubrir una brecha para los investigadores de habla inglesa, con el valor agregado de analizar en inglés componentes claves de los estudios culturales sobre Paraguay. A futuro, el libro podría traer cambios en los estudios paraguayos. Sin embargo, urge que también sea publicado en español, la experiencia de la lectura y comentarios con otros colegas así lo considera. El texto, que puede inscribirse en los estudios culturales, contiene entendimientos que podrían considerarse interdisciplinarios.

El primer aprendizaje sería que es claramente posible, con rigurosidad y con ciencia, hablar sobre Paraguay sin ser paraguayo, aún en temas que eventualmente requieran perspectivas situadas. El libro fue producido a instancias de la academia norteamericana con participación de algunos autores paraguayos. Puede haber matices y complementos al contenido, pero no se puede desmerecer la calidad y rigurosidad de esta obra y su valor contributivo: Paraguay ha sido históricamente olvidado en los estudios académicos regionales y mundiales. Hay causas y factores para ello, siendo el principal el rezago del desarrollo académico, científico e investigativo en Paraguay, lo cual está cambiando en los últimos cinco años, por lo tanto, el momento histórico para la publicación de este libro es ideal.

Surge entonces la primera interrogante. ¿De qué manera se da la aproximación a estudiar Paraguay, siendo el mismo un objeto de estudio poco conocido?, la principal respuesta es que el autor tuvo la oportunidad de estar físicamente cerca del Paraguay, en un momento en el cual estaban ocurriendo situaciones llamativas en la política partidaria del país. Situaciones que eran llamativas por tener rasgos similares, pero a la vez distintivos con relación a la historia latinoamericana de inestabilidades políticas y autoritarismos. Concretamente el golpe de estado al gobierno de Fernando Lugo desencadenó muchas preguntas para la comprensión internacional del Paraguay, y Federico Pous no pudo ser indiferente: había rasgos culturales que debían ser estudiados, el autoritarismo era uno de ellos.

La Exposición de Paraguay está organizada en cuatro secciones que son a la vez ejes de la exposición, el primero de ellos "Writing the Limits of Authoritarian Paraguay" ilustra antecedentes históricos del autoritarismo. La segunda sección, "Preaching Popular Art in Paraguay", probablemente la más desafiante para el lector ajeno al área, contiene las primeras sorpresas novedosas - desde los estudios culturales enfocados en el arte - para los estudios sociológicos y políticos sobre las simbologías existentes en el arte del Paraguay, esta sección propone un criterio necesario para abordar la cuestión de lo local versus lo regional en las expresiones culturales de un país que frecuentemente se autoconcibe como aislado. Luego de una - aparente - pausa al desarrollo del análisis del autoritarismo, la tercera sección propone un giro en la comprensión del sostén cultural del estronismo, Soler muestra que la dictadura de Stroessner estaba tan legitimada culturalmente que hasta casi no necesitaría ser una dictadura. Los mecanismos utilizados para legitimarla son reveladores y sientan las bases para los siguientes capítulos del libro que contienen lo que se consideraría los aportes didácticamente más trascendentes para el lector paraguayo: el análisis de películas contemporáneas famosas y conocidas por la mayoría. 108/Cuchillo de Palo es la primera película paraguaya que trató sobre la historia reciente de las expresiones culturales de la homofobia, en una sociedad altamente conservadora como es la paraguaya, por lo tanto, podría ser un quiebre. 108 es el número de personas de una lista de homosexuales perseguidos por la policía estronista y, a la vez, en Paraguay en la década del ochenta y noventa "108" fue una expresión utilizada de manera corriente para referirse a homosexuales, muchas veces sin saber la historia de tal expresión. El capítulo que analiza la película Siete Cajas ahora puede ser utilizado para cualquier docencia sobre marxismo en Paraguay. La película Siete Cajas monta una película de acción y suspenso sobre los sueños y las historias de un adolescente de clase obrera informal del Paraguay. El capítulo 
retoma dicho punto con una novedosa e inesperada reconstrucción de la película -conocida por la mayoría de los paraguayos - basada en elementos de análisis marxista que con claridad ilustra puntos de vista que han sido pasados por alto en otros análisis hechos sobre la misma película.

La exposición de Paraguay, que también es un viaje, concluye trabajando en la última sección con una expresión llamativa e inquietante de por sí, e inspiradora para todo aquel que se siente obnubilado por los misterios del devenir paraguayo. La denominación de la sección: “Tracing La realidad que delira” (Rastreando la realidad que delira) resume la motivación que subyace al hecho que motivó la redacción del libro, y expresa una humildad de la obra, que logra ofrecer apenas rastros, indiscutibles y excelentemente expuestos, para una comprensión del Paraguay que necesariamente debe ser profundizada. El libro incluye el análisis de otra película paraguaya muy famosa llamada Hamaca Paraguaya, una película cuyos recursos estéticos, narrativos y lingüísticos son desafiantes para el público paraguayo. Esta película es una revisión de la guerra del chaco, con Bolivia, desde una pareja de padres de un soldado que ha ido a la guerra, expone diversos símbolos culturales de la percepción de dicho evento histórico. Todas las películas analizadas en el libro son indicadores de una incipiente pero creciente industria cinematográfica en Paraguay, y todas las películas representan un quiebre en cada estreno. De momento, es la primera obra académica que aborda estos elementos modernos claves para la comprensión del Paraguay.

El libro culmina con un ensayo que parece saludar a Rafael Barret, quien llegó a Paraguay a integrarse interculturalmente, pues contribuyó enormemente a su comprensión, de manera similar a lo que hace este libro. Uno de los principales aportes de esta exposición de Paraguay, es que puede ser un primer paso hacia entre el uso de la expresión "La realidad que delira" que proviene de la literatura, como una categoría científica, para los estudios académicos sobre Paraguay. Sin duda, dicha expresión, tras profundizarla más puede ser un puente entre el conocimiento literario y el conocimiento científico.

Esta realidad que delira, expresión tal vez relacionable al realismo mágico "se refiere a un espacio y un tiempo que existe dentro de dos márgenes: por un lado, la realidad de la injusticia social y, por otro, la reactivación de los afectos por los sentimientos apasionados" (p. 270). La realidad que delira es una frase que estaría haciendo referencia a una realidad fantasiosa, esa situación resuena y parece ponerle nombre a algo que cualquier sujeto curioso y conocedor del Paraguay sabe, aquella característica de la cultura paraguaya de tender a ser inconsciente de la realidad o ciegamente optimista.

El libro representa una ubicación del Paraguay en los estudios latinoamericanos y en la ciencia internacional. El autoritarismo como rasgo cultural paraguayo es un factor importante, y de apariencia omnipresente para la comprensión del país, pero no es el único: lo que intenta expresarse con la realidad que delira también es un rasgo principal del Paraguay. La obra es brillante por ofrecer un viaje desde aspectos históricos clásicos hasta expresiones artísticas recientes, pasando por análisis del arte paraguayo, y ofreciendo entendimientos que representan anclajes para los estudios de género, estudios sociológicos, politológicos. Frecuentemente se considera que Paraguay es un país aislado, que posee traumas bélicos e históricos y con poca resistencia colectiva basada en poca memoria histórica. Este libro propone un viaje que revisa y relativiza cada uno de los lugares comunes que existen sobre Paraguay y llega a un punto que puede ser inicio para otro viaje con aquello de la realidad que delira.

El libro exitosamente integra la particularidad de Paraguay en una matriz conceptual más amplia, ofrece respuestas sobre rasgos culturales autoritarios del Paraguay, y contribuye a plantearse nuevas interrogantes tal vez mas refinadas que las iniciales antes de leer el libro, sobre las similitudes y diferencias del devenir paraguayo, desde un punto de vista cultural. 